\title{
Spontaneous Bowel Perforation - A Rare and Fatal Complication in COVID-19 Patients: Case Report
}

\author{
Lamya Zaher Al Aamri', Mohammed Muzamil ${ }^{1}$, Nasser Alawaid ${ }^{1}$, \\ Pratap Kumar Shinde ${ }^{2}$ \\ ${ }^{1}$ Department of Medicine, Sultan Qaboos Hospital, Salalah, Sultanate of Oman \\ ${ }^{2}$ Department of Surgery, Sultan Qaboos Hospital, Salalah, Sultanate of Oman \\ Corresponding Author: Lamya Zaher Al Aamri
}

\begin{abstract}
Coronavirus 2019-nCoV (COVID-19) is a global pandemic and highly infectious disease, with most of the symptoms being related to the respiratory tract. However, more extrapulmonary symptoms and complications are evolving and directly associated with COVID- 19 pathogenesis. A recognized clinical manifestation of COVID-19 is gastrointestinal and hepatic involvement. We reported a middle-aged man admitted to the intensive care unit (ICU) with severe COVID-19 pneumonia; he developed spontaneous bowel perforation. He had not received tocilizumab as the latter was reported to be associated with bowel perforation.
\end{abstract}

Keywords: Coronavirus 2019-nCoV (COVID-19, bowel perforation

\section{INTRODUCTION}

Since December 2019, the global pandemic caused by the highly infectious novel coronavirus 2019-nCoV (COVID-19) has been rapidly spreading. ${ }^{1}$ The outbreak has spread worldwide, with millions of confirmed cases. Oman is one of many countries severely affected by COVID- 19 .

Respiratory symptoms are the most clinical manifestation of COVID-19, like fever, cough, shortness of breath, and ARDS. Extrapulmonary involvement by SARS-CoV-2 infection has been known and recognized. The extrapulmonary clinical manifestation includes hepatitis, acute renal failure, encephalitis, myocardial injury, gastroenteritis, and coagulopathy. ${ }^{2}$

Here we report a case of a patient who confirmed COVID- 19 positive by RTPCR and a developed bowel perforation.

\section{CASE REPORT}

A 64-year-old man presented to the emergency department on 11th Sep 2020 with four days of cough, fever, and shortness of breath. His Past medical background included type 2 Diabetic Mellitus, hypertension, ischemic heart disease, and dyslipidemia. Clinically, he was hypoxia $91 \%$ room air, afebrile $36.7^{\circ} \mathrm{C}$, weight $83 \mathrm{Kg}$, BMI 30.5. On examination, he was tachypnea at a rate of 25 breaths per minute; chest auscultation revealed bilateral crackles with good air entry, no sign of effusion or lung collapse, and the trachea was central. Cardiac examination showed no galloping or murmur, and there was no lower limb pitting edema. His abdominal exam was normal with no organomegaly and normal audible bowel sounds.

Blood investigation revealed a white blood count of $9.57 \times 10^{3} / \mathrm{ul}$, hemoglobin of $12.72 \mathrm{~g} / \mathrm{dl}$, hematocrit of $43.04 \%$, platelet count of $209 \times 10^{3} / \mathrm{ul}, \mathrm{C}$ reactive protein of $236.62 \mathrm{mg} / \mathrm{l}$, ferritin of $1150 \mathrm{ng} / \mathrm{ml}$, lactate dehydrogenase of $545 \mathrm{u} / 1$ and troponin of $0.016 \mathrm{ng} / \mathrm{ml}$. His chest $\mathrm{X}$-ray showed predominantly bilateral diffuse patch 

report.

opacities. Subsequently, SARS Cov-2 using polymerase chain reaction resulted in positive. Initially, He had received a usual COVID care according to the local hospital protocol, which included frusemide, methylprednisolone, heparin, and ceftriaxone empirically waiting for cultures. On the third day of admission, his respiratory status progressed, and he initially needed a non-rebreather mask. Eventually, he required high oxygen flow nasal cannula (HFNC), upgraded to piperacillin-tazobactam, and received one unit of convalescent plasma. The Next day, his ventilator support upgraded to noninvasive ventilation (NIV) as his oxygen requirement increased.

$\mathrm{He}$ received a second unit of convalescent plasma according to the protocol after 48 hours of the initial unit. In the following few days, the patient respiratory condition improved, and ventilation requirements reduced.

On the 9th day, he developed abdominal distension and pain. He denied any heartburn, nausea, or vomiting. As a result, an abdominal $\mathrm{x}$-ray was requested and showed bowel loop dilatation (Fig.1). The surgery team consulted initially advised conservative management as likely to be ileus. On the 14th-day patient's general condition worsened with increased abdominal distension with bowel movement despite laxative, and his inflammatory markers went very high. He required vasopressor support to maintain his blood pressure. An urgent abdominal CT scan was obtained and demonstrated massive pneumoperitoneum compressing the abdomen and elevates the diaphragm, compressing the chest along with air-fluid level at the right subphrenic region (Fig.2).

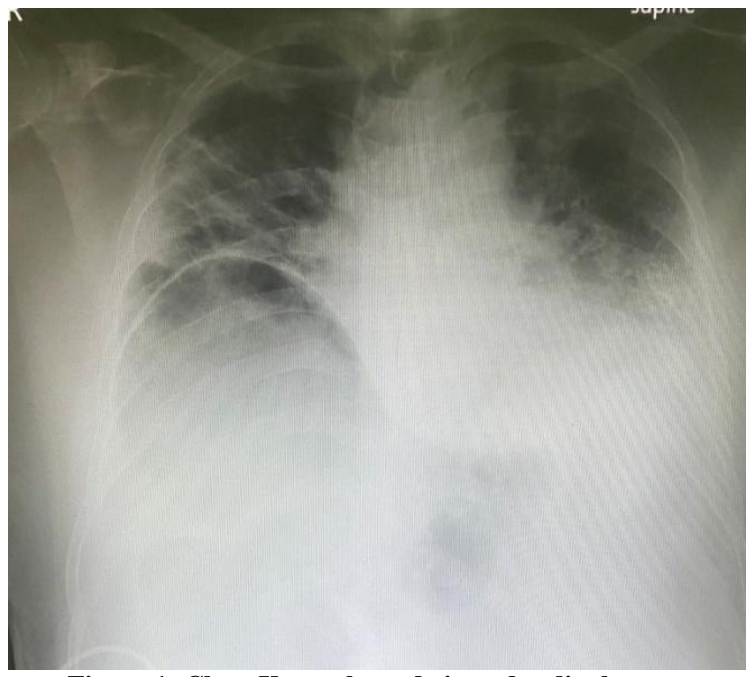

Figure 1: Chest X-ray showed air under diaphragm.
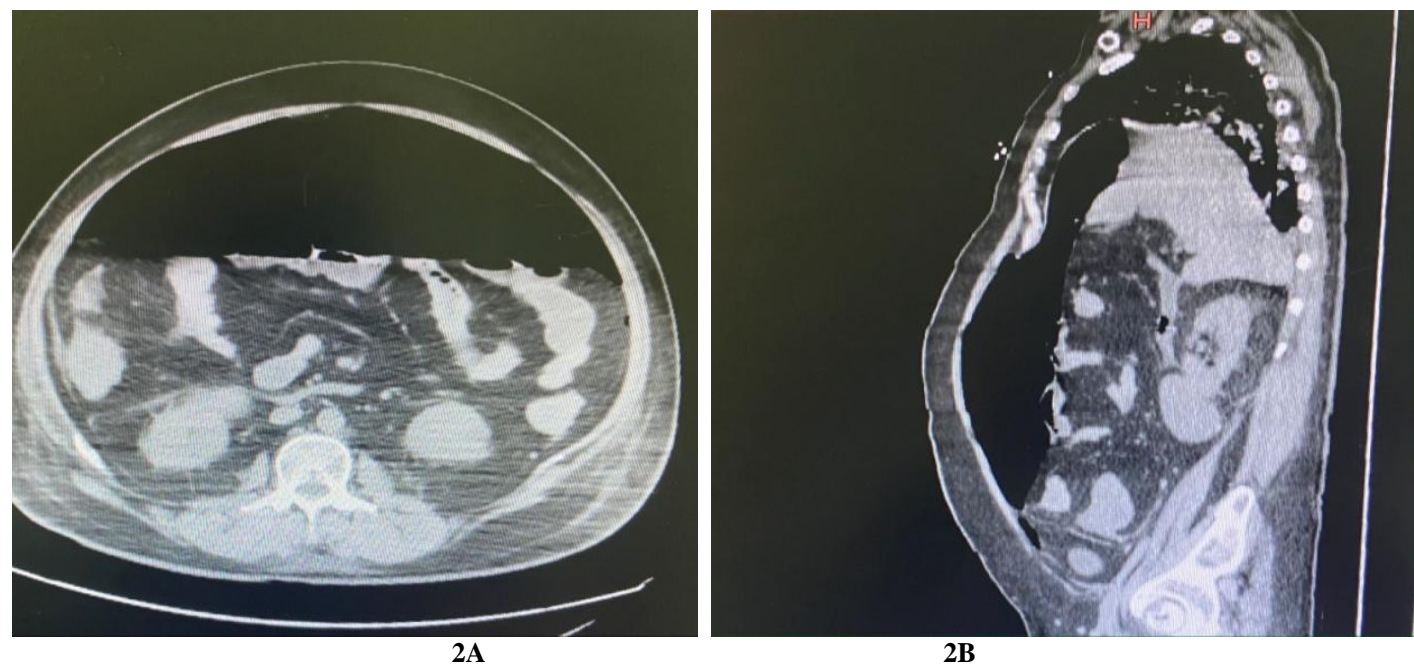

Figure 2: Computed Tomography (CT) show pneumoperitoneum, 2A: axial view and 2B sagittal view

The patient was taken urgently to the operating room for exploration and subsequently intubated. Upon exploration, found to have two large perforations near the proximal part of the transverse colon with gross contamination of the abdominal cavity. In addition, there were multiple flakes present over the caecum ascending transverse and sigmoid colon. 

report.

The histopathology reported a hemorrhage and inflammatory exudates in the lumen. In addition, there was mucosal ulceration, perforation, and transmural infiltration by lymphocytes, histiocytes, eosinophils, and few neutrophils. Also, there was serositis and fibrinoid necrosis.

He required mechanical intubation and remained in critical condition. On the 18th-day patient developed extensive subcutaneous surgical emphysema, resolved with conservative management. Unfortunately, he died one week later.

\section{DISCUSSION}

Extrapulmonary manifestation of COVID 19 is recognized. Approximately up to $50 \%$ of COVID-19 patients have gastrointestinal symptoms. Most common symptoms, Loss of appetite and diarrhea and less often vomiting and abdominal pain. ${ }^{3}$ COVID-19 virus enters enteric epithelial tissue through ACE 2 and transmembrane protease, serine $2 .{ }^{4}$ ACE2 highly expression in the lung alveolar epithelium, enterocytes of the small intestine, and vascular endothelium. Indicate a SARS-CoV-2 infection could be susceptible to infect the bowel and vasculature $^{.5,6}$

Demonstrated SARS-CoV-2 RNA in the cytoplasm of gastrointestinal epithelial cells and the stool. ${ }^{4,7}$

There were a few reports of suggestive bowel perforation related to the COVID-19 virus. In our case patient was in ICU developed bowel perforation during hospitalization for COVID-19. Images confirmed pneumoperitoneum and treated initially conservative; however, he deteriorated and needs urgent operation.

Rajesh et al. ${ }^{8}$ By reviewed 412 reports of patients demonstrate a $31 \%$ of bowel-wall abnormalities were in CT images and were associated with ICU admission. In addition, pneumatosis or portal venous gas was in $20 \%$ of bowel images. The surgical finding revealed unusual yellow discoloration of the bowel and bowel infarction. Furthermore, pathologic showed ischemic enteritis with patchy necrosis and fibrin thrombi in arterioles.

In our patient, CT images could not appreciate any bowel wall abnormalities due to massive pneumoperitoneum.

The pathophysiology mechanism of perforation in COVID could be: 1 . A direct injury direct insult to the colonic cells by the coronavirus 2. An autonomic innervation injury of the colon leads to affect colonic motility 3. hypercoagulable state in COVID19 lead to intravascular coagulation, which manifests as generalized small-vessel vasculitis and extensive microthrombi. ${ }^{9,10}$

Studies demonstrate that tocilizumab and accumulative glucocorticoid are participating risk factors in developed bowel perforation. $^{11}$

Our patient had never received tocilizumab. He was on a short course of methylprednisolone as follow local guidelines. Consider SARS-Cov-2 infection the only reason for bowel perforation.

\section{Acknowledgement: None}

\section{Conflict of Interest: None}

\section{Source of Funding: None}

\section{REFERENCES}

1. Lu H, Stratton cW, Tang Y-W. Outbreak of pneumonia of unknown etiology in Wuhan, china: the mystery and the miracle. J Med Virol 2020;92:401-2

2. Zhu N, Zhang D et al (2020) A novel coronavirus from patients with pneumonia in China, 2019. N Engl J Med 382:727733. https://doi.org/10.1056/NEJMoa2001017

3. Kotfis K, Skonieczna-Żydecka K (2020) COVID-19: gastrointestinal symptoms and potentialCOVID-19: gastrointestinal symptoms and potential sources of 2019$\mathrm{nCoV}$ transmission. Anaesthesiol Intensive 52:1. https://doi.org/10.5114/ait.2020.93867

4. Jin X, Lian J-S, Hu J-H. Epidemiological, clinical and virological characteristics of 74 cases of coronavirus-infected disease 2019 (COVID-19) with gastrointestinal symptoms. Gut. 2020;0:1-8. doi: 10.1136/ gutjnl-2020-320926 
5. Zou X, Chen K, Zou J, Han P, Hao J, Han Z. Single-cell RNA-seq data analysis on the receptor ACE2 expression reveals the potential risk of different human organs vulnerable to $2019-\mathrm{nCoV}$ infection. Front Med 2020;14(2):185-192.

6. Hamming I, Timens W, Bulthuis MLC, Lely AT, Navis G, van Goor $\mathrm{H}$. Tissue distribution of ACE2 protein, the functional receptor for SARS coronavirus. A first step in understanding SARS pathogenesis. J Pathol2004;203(2):631-637.

7. Tian Y, Rong L, Nian W, He Y. Review article: gastrointestinal features in COVID19 and the possibility of faecal transmission. Alimentary Pharmacology \& Therapeutics. 2020;51(9):843-851. doi: 10.1111/apt.15731

8. Rajesh B, Avik s, Matthew D.L at el. bdominal Imaging Findings in COVID-19: Preliminary Observations. Radiology 2020; 297:E207-E215. https://doi.org/10.1148/radiol.2020201908

9. Y. Song, P. Liu, X.L. Shi, Y.L. Chu, J. Zhang, J. Xia, et al. SARS-CoV-2 induced diarrhoea as onset symptom in patient with
COVID-19.Gut, (2020), http://dx.doi.org/ 10.1136/gutjnl-2020-320891

10. Zhou B, She J, Wang Y, Ma X. Venous thrombosis and arteriosclerosis obliterans of lower extremities in a very severe patient with 2019 novel coronavirus disease: a case report. J Thromb Thrombolysis. 2020, http://dx.doi.org/10.1007/s11239-02002084-w

11. Trangfeld A, Richter A, Siegmund B, Herzer P, Rockwitz K, Demary W. Risk for lower intestinal perforations in patients with rheumatoid arthritis treated with tocilizumab in comparison to treatment with other biologic or conventional synthetic DMARDs. Ann Rheum Dis. 2017;76:504510. doi: 10.1136/annrheumdis-2016209773

How to cite this article: Lamya Zaher Al Aamri, Muzamil M, Alawaid N et.al. Spontaneous bowel perforation - a rare and fatal complication in COVID-19 patients: case report. Int J Health Sci Res. 2021; 11(8): 198-201. DOI: https://doi. org/10.52403/ijhsr.20210829 